\title{
Determining the Dynamic Balance, Maximal Aerobic Capacity, and Anaerobic Power Output of University Soccer and Rugby Players: A Cross-Sectional Comparative Study
}

\author{
Mohammad Ahsan ${ }^{1}$, Mohammad Feroz Ali ${ }^{2, *}$ \\ ${ }^{1}$ Department of Physical Therapy, College of Applied medical Sciences, Imam Abdulrahman Bin Faisal University, \\ Dammam, Saudi Arabia \\ ${ }^{2}$ Department of Secondary and Sports Education, College of Humanities and Education, Fiji National University, \\ Lautoka Campus, Lautoka, Fiji
}

Received September 26, 2021; Revised November 23, 2021; Accepted November 27, 2021

\section{Cite This Paper in the following Citation Styles}

(a): [1] Mohammad Ahsan, Mohammad Feroz Ali , "Determining the Dynamic Balance, Maximal Aerobic Capacity, and Anaerobic Power Output of University Soccer and Rugby Players: A Cross-Sectional Comparative Study," International Journal of Human Movement and Sports Sciences, Vol. 9, No. 6, pp. 1486 - 1492, 2021. DOI: 10.13189/saj.2021.090646.

(b): Mohammad Ahsan, Mohammad Feroz Ali (2021). Determining the Dynamic Balance, Maximal Aerobic Capacity, and Anaerobic Power Output of University Soccer and Rugby Players: A Cross-Sectional Comparative Study. International Journal of Human Movement and Sports Sciences, 9(6), 1486 - 1492. DOI: 10.13189/saj.2021.090646.

Copyright $\bigcirc 2021$ by authors, all rights reserved. Authors agree that this article remains permanently open access under the terms of the Creative Commons Attribution License 4.0 International License

\begin{abstract}
Physical performance characteristics of players have been changed rapidly as team games have become more competitive. Research focusing on the anthropometrical and physiological characteristics of players and the demand of competition are reviewed. The dearth of research for today's elite players was highlighted, along with the need for standardized testing protocols. This research was conducted to investigate the differences in physical performance characteristics between soccer and rugby players with standardized testing protocols. A total of thirty-six male subjects (18 soccer players and 18 rugby players) were chosen for this study. The subjects had an average age of $20.50 \pm 1.36$ years, a height of $175.28 \pm 5.554 \mathrm{~cm}$, weight of $67.73 \pm 6.47 \mathrm{~kg}$, and BMI of $22.01 \pm 1.01$. Any player who had a musculoskeletal injury in their lower extremity or had a record in the previous three months was excluded from the research. Dynamic balance was determined using the Y Balance Test. The Beep test assessed maximal aerobic capacity, and the vertical jump test evaluated the anaerobic power output of the lower body. An independent t-test was applied to determine the differences between soccer and rugby players. Our finding showed that there were insignificant
\end{abstract}

differences for dynamic balance $(\mathrm{t}=-1.31, \mathrm{p}=.19)$, maximal aerobic capacity $(\mathrm{t}=.94, \mathrm{p}=.35)$, and anaerobic power output $(\mathrm{t}=-.403, \mathrm{p}=.69)$ between soccer and rugby players. Findings are also evident for the decrease in anaerobic performance with increased maximal oxygen capacity in soccer players while anaerobic performance increases and maximal aerobic capacity decreases in rugby players. Functional tests for performance parameters should be considered when planning to train players, predict performance, and identify sports talent.

Keywords Dynamic Balance, Aerobic Capacity, Anaerobic Power, Beep Test, Y-Balance Test

\section{Introduction}

Soccer and rugby are the world's most famous sports because of their gargantuan acceptance. These field-based team games are intermittent high-intensity sports eliciting several physical performance parameters such as high-intensity sprints, acceleration, cutting movements, 
sidestepping, jumping, tackling, specific technical and tactical skills [1]. According to studies, $80-90$ percent of these sports involve high-intensity work [2]. Players should have the appropriate amount of strength, endurance, power, speed, acceleration flexibility and balance [3], which meet the pre-requirements of both sports.

Dynamic balance is a fundamental skill that is implicated in the multiple scenarios of sports activities. Dynamic balance is not just essential to participate in sports activities, it can also be a predictor of injury. Dynamic balance deficits have been studied extensively as a predictor of lower extremity injury [4]. A study revealed that players with lift-to-right leg balance discrepancies or with poor balance were 3.86 time more likely to sustain a lower extremity injury [5]. On this issue, Ringhof and Stein elaborated on the traditional perspective of balance as a broad skill and reaffirmed the need of dynamic balance testing [6]. The Y-balance test is a common, reliable, and simple balance evaluation technique. Studies have shown a connection between Y-balance test score and possible lower limb injuries in diverse populations $[7,8]$. According to Johnston et al., poor dynamic balance as evaluated by Y-balance test increases the relative risk of sports-related injuries among rugby union players [9]. This test has been shown very beneficial in a wide range of applications, including many in sports and rehabilitation processes, as well as in the evaluation of children, adolescents, and adults $[10,11]$.

A high level of aerobic and anaerobic fitness is required to participate in soccer and rugby games. Throughout the game, players must be able to perform repeated maximal or sub-maximal intensive activities that require a high oxidative energy level. According to research findings, soccer players' aerobic capacity is affected by their level of competitiveness. [12]. As a result, measures of maximum oxygen consumption are the best measure to assess the maximal aerobic capacity and might be used to identify a successful player or team [13]. Maximal aerobic capacity can be measured by the field or laboratory testing methods. Beep test, also known as the multistage, entails sprinting at different speeds across a 20-meter distance, integrated with frequent dynamic twisting and change of direction, which is said to be unique to soccer and rugby movement [14]. The performance during the beep test has been validated with measure of maximal aerobic capacity obtained during treadmill running test [14]. Leger et al. conclude that beep test is a good predictor of maximal aerobic capacity. They provide an equation based on the maximum speed at the test run for estimation of maximal aerobic capacity [15].

Anaerobic performance is characterized by explosive workouts that are completed in a short period of time. Whereas, in case of prolonged muscular activity, source of energy is initiated through anaerobic processes followed by an aerobic process [16]. Explosive power and endurance power stated as the ability should keep as much power as possible for as long as possible. These anaerobic components of energy capacity may be tested in a variety of methods, with varying degrees of accuracy. [17]. The vertical jump test is the most popular way to assess anaerobic power output. The most common prediction equations used with the vertical jump is Lewis formula [18]. Maccurdy et al. recognized explosive movement such as short sprint and vertical jump as major predictors of anaerobic power output. These variables are responsible for more than $50 \%$ of the variance in the performance measures [19]. Many studies have been accomplished in an attempt better to understand performance by the players. However, since each sports team has different characteristics such as the number of players, playing duration, space, equipment used, etc., these might be reasons for players' differences in physical fitness components. It is expected that soccer and rugby players are different from each other. Therefore, this study aimed to investigate the physical performance characteristics differences among soccer and rugby players with standardized testing protocols. We hypothesized that soccer and rugby players would have significant differences and significant differences also exist among physical performance parameters.

\section{Materials and Methods}

\subsection{Experimental Design}

The current study employed a comparative cross-sectional design to measure and compare physical performance parameters between university soccer and rugby players. This study was conducted using a probability sampling approach. Only those participants who want to partake in this study willingly and fulfil all of the inclusion and exclusion criteria were chosen.

\subsection{Participants}

A total of thirty-six male subjects (18 soccer players and 18 rugby players) were chosen for this study. Eighteen subjects in one group were deemed sufficient for establishing a difference between two groups with sufficient power (80\% at p 0.05) [20]. All of the subjects were university players with at least two years of experience at this level. Before the research, all participants were informed of the study's aim and the related experimental hazards. The subjects had an average age of $20.50 \pm 1.36$ years, a height of $175.28 \pm 5.554 \mathrm{~cm}$, weight of $67.73 \pm 6.47 \mathrm{~kg}$, and a BMI of $22.01 \pm 1.01$. Any player who had a musculoskeletal injury in their lower extremity or had a record in the previous three months was excluded from the research. 


\subsection{Anthropometric Measurements}

\subsubsection{Height}

Heights of subjects were measured by using a standardized stadiometer. The measurement was taken to the nearest of $0.1 \mathrm{~cm}$.

\subsubsection{Weight}

Weights of the subjects were measured by using an auto-calibrated weighing scale.

\subsubsection{Body Mass Index}

Body Mass Index (BMI) is calculated as weight (in kilograms) divided by the square of height (in meters) or $\mathrm{BMI}=\mathrm{Kg} / \mathrm{M} 2$.

\subsection{Physical Performance Parameters}

\subsubsection{Dynamic Balance}

Dynamic balance was assessed using the Y Balance Test (YBT). The YBT is a functional test that assesses dynamic balance in three directions: anterior, posteromedial, and posterolateral. The interrater test-retest reliability for the YBT is good $(\mathrm{ICC}=0.80-0.85)$ [21].

\subsubsection{Maximal Aerobic Capacity}

The Beep test was used to determine a child's, adolescent's, or adult's maximum aerobic capacity. The 20-meter shuttle run test is also known as the beep test. In a field setting, the test gives a realistic and cost-effective prediction of maximal aerobic capacity. Participants were instructed to run in a shuttle style toward and away from 20-meter portions in response to an auditory signal (beep) provided by an audio player. The initial running pace was $8.5 \mathrm{~km} / \mathrm{hr}$, and the pace increased by $0.5 \mathrm{~km} / \mathrm{hr}$ per minute. This increased running pace was described as a change in test level. Before the next beep sounded, participants had to finish a level.

\subsubsection{Anaerobic Power Output}

The vertical jump test was used to determine the lower body's anaerobic power output. Dr Dudley Allen Sargent developed the vertical jump test in 1921 to measure leg muscle power [22]. Subjects took part in a vertical leap test using Vertec-designed equipment. This test is simple and quick to perform.

\subsubsection{Equipment}

To measure anthropometric characteristics of subjects- a stadiometer and a weighing scale were used. The YBT was conducted using a $2 \times 2$ meter free and reliable space, adhesive tape, measurement tape, and performance recording sheets. The following standardized equipment was used to conduct the beep test: a 20 -meter shuttle run space, cones, metronome, and performance recording sheets. A Vertec was used to measure anaerobic power.

\subsubsection{Procedure}

Before the subjects came, the Y balance platform and the 20-meter shuttle run space were designated. A part of space was assigned for the vertical jump test. Upon the subjects' arrival at the university indoor hall, researchers took the subjects' anthropometric measurements and asked them to fill a sociodemographic questionnaire. The dominant limb of each subject was identified by asking which leg they utilized to kick a ball [23]. Throughout the testing, all the subjects wore sports-specific attire. Before the start of the test, participants were given a ten-minute warm-up on an exercise bicycle. The YBT was done on a test platform marked in three directions: anterior, posterolateral, and posteromedial. The subject might have practice trials in each direction with their dominant leg. The YBT test was performed to stand barefoot on the centre platform and await further instructions from the researcher. Participants were asked to reach with a free leg in the anterior direction three times, then the posterolateral direction three times, and finally the posteromedial direction three times while standing on one leg. The maximal reach distance was recorded in all trials. A trial was discarded and repeated if the participant lost his balance, lifted his heel off the platform, and took any support. The sum of maximal reach distance in each direction was divided by three trials to yield an absolute reach distance, which was used to analyze the overall performance of the YBT test. The scores of the dominant leg were used for further data analysis.

The Beep test consisted of subjects running in a shuttle format towards and away from a 20-meter section in response to an auditory signal (beep) produced by a metronome (audio player). The pace was $8.5 \mathrm{~km} / \mathrm{hr}$ in the first minute and rose by $0.5 \mathrm{~km} / \mathrm{hr}$ every minute after that. Before the next beep sounded, subjects had to complete a level. Subjects were expected to complete as many shuttles as possible. If a participant fails to maintain the required pace for two consecutive shuttles or withdraws from the test owing to fatigue, the test will be terminated. The maximum aerobic capacity was calculated using the method proposed by Flouris et al. [24]:

$$
\begin{gathered}
\text { Maximal Oxygen Capacity }(\mathrm{mL} / \mathrm{min} / \mathrm{kg})=(\max . \text { attained } \\
\text { speed }(\mathrm{km} / \mathrm{h}) \times 6.65-35.8) \times 0.95+0.182
\end{gathered}
$$

To measure the anaerobic power of lower leg muscles vertical jump test was used. The subject must first push aside as many vanes as possible while standing flat-footed to measure the vertical jump with a Vertec. The standing reach height was computed in this manner. The subject then stands beside the Vertec and jumps to the maximum possible vertical height, utilizing arms and legs to assist the body to push higher in a countermovement. Try pushing aside many extra vanes of the Vertec device while jumping. The difference between the standing reach height and the height of the jump was used to calculate the score. The scores were converted to the units of power through the established equation for peak power developed by Johnson 
\& Bahamonde Formula as below [25].

Peak power $(\mathrm{W})=78.5 \times \mathrm{VJ}(\mathrm{cm})+60.6 \times$ mass $(\mathrm{kg})$ $-15.3 \times$ height $(\mathrm{cm})-1308$

All the tests were conducted using standard procedure and instructions [24-26].

\subsubsection{Statistical Analysis}

IBM SPSS (V. 26) was used for all statistical analyses. The data were verified for normality and outliers before being analyzed. The data were presented in the form of mean values and standard deviation (SD). A t-test was conducted for age, height, weight, and BMI. For physical performance parameters (Dynamic Balance, Maximal Aerobic Capacity, and Anaerobic Power), an independent t-test was also done comparing soccer and rugby players. An $\alpha$-level of 0.05 was used to determine statistical significance.

\section{Results}

All anthropometric parameters, data normality, and differences of variables (dynamic balance, maximal aerobic capacity, and anaerobic power) between soccer and rugby players were examined. Insignificant differences have been seen in weight $(\mathrm{p}=.698)$ and height $(\mathrm{p}=.234)$. The data were normally distributed, and insignificant differences for dynamic balance $(\mathrm{t}=-1.31, \mathrm{p}=.19)$, maximal aerobic capacity $(\mathrm{t}=.94, \mathrm{p}=.35)$, and anaerobic power $(t=-.403, p=.69)$ can be seen. Therefore, it is clear that the hypothesis for this study was not accepted, as there were no significant differences between dynamic balance, maximal aerobic capacity, and anaerobic power for soccer and rugby players.

Table 1 showed significant differences between soccer and rugby player's anthropometric characteristics in terms of age $(\mathrm{p}=.049)$ and BMI $(\mathrm{p}=.015)$. In contrast, no significant differences have been seen in weight $(\mathrm{p}=.698)$ and height ( $\mathrm{p}=.234$ ), with a significant level at 0.05 .

Table 2 showed that the statistic values are close to 1 , and no significant differences were found in soccer and rugby players. Therefore, we assume that data for dynamic balance, maximal aerobic capacity, and anaerobic power were normally distributed, and further analysis was done using the parametric tests.

Table 1. Participant's anthropometric characteristics and body composition

\begin{tabular}{|c|c|c|c|c|c|}
\hline & Soccer Mean \pm SD & Rugby Mean \pm SD & Std. Error & t & $\begin{array}{c}\text { Sig. } \\
\text { (2-tailed) }\end{array}$ \\
\hline AGE & $20.94 \pm 1.47$ & $20.06 \pm 1.11$ & .347 & 2.044 & $.049 *$ \\
\hline WEIGHT & $67.30 \pm 6.96$ & $68.16 \pm 6.12$ & 1.64 & -.392 & .698 \\
\hline HEIGHT & $176.39 \pm 4.32$ & $174.17 \pm 6.46$ & 1.020 & 1.212 & .234 \\
\hline BMI & $21.61 \pm 1.19$ & $22.41 \pm .59$ & 0.28 & -2.561 & $.015^{*}$ \\
\hline
\end{tabular}

*Significant at 0.05 level

Table 2. Test of normality in Dynamic Balance, Maximal Aerobic Capacity, and Anaerobic Power for soccer and rugby players

\begin{tabular}{|c|l|c|c|c|c|c|}
\hline \multirow{2}{*}{ Sports } & \multicolumn{2}{c|}{$95 \%$ CI } & \multicolumn{3}{c|}{ Shapiro-Wilk } \\
\cline { 3 - 8 } & & Lower & Upper & Statistic & df & Sig. \\
\hline \multirow{2}{*}{ Dynamic Balance } & Soccer & 35.79 & 39.08 & .971 & 18 & .810 \\
\cline { 2 - 8 } & Rugby & 37.31 & 40.36 & .913 & 18 & .098 \\
\hline \multirow{2}{*}{\begin{tabular}{c} 
Maximal Aerobic Capacity \\
\cline { 2 - 8 }
\end{tabular}} & Soccer & 59.76 & 70.80 & .962 & 18 & .376 \\
\cline { 2 - 8 } & Rugby & 56.94 & 66.93 & .983 & 18 & .443 \\
\hline \multirow{2}{*}{ Anaerobic Power } & Soccer & 447.68 & 719.10 & .956 & 18 & .527 \\
\cline { 2 - 8 } & Rugby & 444.61 & 809.07 & .973 & 18 & .264 \\
\hline
\end{tabular}

Table 3. Differences between soccer and rugby university players for dynamic balance, maximal aerobic capacity, and anaerobic power

\begin{tabular}{|c|c|c|c|c|c|}
\hline & Soccer Mean \pm SD & Rugby Mean \pm SD & Std. Error & $\boldsymbol{t}$ & $\begin{array}{c}\text { Sig. } \\
\text { (2-tailed) }\end{array}$ \\
\hline Dynamic Balance & $37.44 \pm 3.31$ & $38.84 \pm 3.07$ & 0.78 & -1.313 & .198 \\
\hline Maximal Aerobic Capacity & $65.28 \pm 11.09$ & $61.94 \pm 10.04$ & 2.62 & .948 & .350 \\
\hline Anaerobic Power & $583.39 \pm 272.90$ & $626.84 \pm 366.45$ & 64.32 & -.403 & .689 \\
\hline
\end{tabular}


Table 3 showed that soccer and rugby players have insignificant differences for dynamic balance $(\mathrm{t}=-1.31$, $\mathrm{p}=.19)$, maximal aerobic capacity $(\mathrm{t}=.94, \mathrm{p}=.35)$ and anaerobic power. $(\mathrm{t}=-.403, \mathrm{p}=.69)$. Whereas the mean scores showed differences for dynamic balance, maximal aerobic capacity, and anaerobic power, these differences are not statistically significant at 0.05 level of significance.

\section{Discussion}

This study aimed to analyze the differences between soccer and rugby players for dynamic balance, maximal aerobic capacity, and anaerobic power. The dynamic balance was better in soccer players $(37.44 \pm 3.31)$ than rugby players $(38.84 \pm 3.07)$, soccer players $(65.28 \pm 11.09)$ have higher maximal aerobic capacity than rugby players $(61.94 \pm 10.04)$, and anaerobic power's mean scores are better in rugby players $(626.84 \pm 366.45)$ than soccer players (583.39 \pm 272.90$)$.

One of the essential skills in soccer and rugby is dynamic balance, which is described as the individual's capacity to complete a task. At the same time, maintain the stability of the centre of mass while moving, usually with a single leg support basis. The static and dynamic balance is determined by the various functional and non-functional tests. Bressel et al. showed that when soccer players are compared to gymnasts and dancers, they have poor dynamic balance. However, they have better balance when compared to basketball players and control participants in the study [27]. Paillard and Noe stated that a higher degree of competitiveness was related to a stronger dynamic balance among soccer players and across different levels of competition [28]. Rugby players use their physical characteristics to conduct open and closed kinetic chain motions to complete game activities and multidirectional running duties that may require enhanced dynamic balance. In a study, rugby players demonstrated better dynamic balance than male American football players and high school mixed-gender basketball players [29]. In our study, soccer players showed better dynamic balance than rugby players. All of these sports need a considerable measure of multidirectional movement as well as postural stability. These differences might be related to the athletes' anthropometric profile, developmental stage, sports-specific demands, and the various test protocol variations that restrict these comparisons. A review of the literature found evidence that there are variations in balancing ability between sports and across performance levels within a specific discipline. Injuries can also be caused by a poor dynamic balance [30,31]. According to the findings of a recent study, rugby players may be at a higher risk of injury than soccer players due to poor dynamic balance.

Physical fitness in any dynamic sports is heavily dependent upon maximal aerobic capacity. A professional player covers a total distance between 10 to $13 \mathrm{~km}$ [32]. Elite soccer players' mean maximum aerobic capacity was in the range of 55 to $65 \mathrm{ml} . \mathrm{kg}-1 . \mathrm{min}-1$. [33]. These values are quite low compared to elite endurance athletes in other sports such as rowing, cycling, or running [34]. According to a research, maximum aerobic capacity did not distinguish soccer players from various levels of competition, including the national team, second division, and juniors. Only the third and fifth division players varied substantially from the other groups in terms of mean maximum aerobic capacity, which was between 61 and $64 \mathrm{mLkg}-1$. min-1. [35]. Ahmadi et al. informed that differences in the Maximal aerobic capacity, as measured by several tests, may be linked to biomechanical characteristics; for example, proper body motions are more effective in terms of energy consumption [36]. In the game of rugby, players play very different roles during the whole game. Maximal aerobic capacity has been established as a key indicator of exercise performance for rugby players. Bellars et al. find a positive and significant association between maximal aerobic capacity and the number of repetitions performed in a timed workout [37]. A study's result indicates that the maximum aerobic capacity was similar in the back and forward rugby players and was lower than the values measured in endurance athletes such as long-distance runners and cyclists [38]. Gabbett stated that maximal aerobic capacity between junior and senior was significantly the same. However, other team players such as Australian football, soccer, and field hockey have maximal aerobic capacity than rugby players [39]. All these findings align with our study as soccer players have a higher amount of maximal aerobic capacity than rugby players. According to $\mathrm{Da}$ Silva et al., an appropriate level of oxygen consumption allows endurance activities to be performed at a higher level of effort or a faster pace. When determining an aerobic capacity profile for soccer players, numerous independent criteria, such as chronological age, biological maturity, and training age, as well as morphology and anthropometrics, must be addressed. [40].

In the research, anaerobic power output was the third most investigated physiological feature among soccer and rugby players. The anaerobic power was measured by using a vertical jump test among soccer and rugby players. The importance of assessing the results of various performance functional tests is well-acknowledged among athletes. Differences in anaerobic power have existed between soccer and rugby players in this current study. Soccer and rugby games include numerous short, explosive exercise bursts followed by shorter recovery intervals over an extended period (90 minutes). During football and rugby contests, aerobic and anaerobic energy systems must be activated to meet muscle energy demands [17]. This study confirms earlier findings on the differences in mean power values elicited throughout the test. While, surprisingly, anaerobic performance decreases 
as playing level increases. Players with higher levels of anaerobic power appear to have lower levels of aerobic power. This might be a disadvantage in terms of enhancing performance, as activities that are vital to the game's outcome frequently need anaerobic performance [41]. Hazzaa et al. reveal that group I had higher anaerobic measures levels, and group II had lower aerobic capacity [42]. Wadley and Le Rossignol observed no significant connection between the anaerobic power and aerobic capacity among Australian base football players [43]. Aziz et al. found no association between aerobic consumption and fastest 40 -meter sprint time, indicating a moderate correlation between aerobic consumption and total time for eight sprints [44]. Minahan et al. evaluated the aerobic capacity and anaerobic power of seven women and seven men. The findings showed that high anaerobic power does not indicate better aerobic capacity. Additionally, there is a relation about the aerobic capacity to protect power problem for 30 seconds bicycle sprint [45]. These aerobic capacities and anaerobic power differences between players may affect the success of teams. High aerobic capacity is primarily responsible for adequate recovery between anaerobic efforts. As a result, players with a better aerobic capacity but a lower anaerobic power may be able to compete well.

\section{Conclusions}

Dynamic balance, maximum aerobic capacity, and anaerobic power have introduced new dimensions to performance standards and are highly valued for soccer and rugby players who demand quick stability, explosiveness, and maximum power production. This research revealed that soccer and rugby players have differences in their performance parameters, but these differences are not statistically significant. Findings also showed that soccer players have lesser anaerobic power than rugby players, but their maximal aerobic capacity is higher than the rugby players. Thus, we can say that those have low level of anaerobic performance their maximal oxygen capacity level is high. In contrast, who have high level of anaerobic performance their maximum aerobic capacity is low. In conclusion, performance parameters for soccer and rugby players such as dynamic balance, aerobic capacity and explosive power must be considered when planning to train players, predict performance, and identifying talent in many sports. In further studies it would be important to identify association of different performance parameters of players in relation to the specific types of sports.

\section{Acknowledgements}

We are very grateful to the Dean and Head of the Department of Physical Education, College of Education and Humanities, Fiji National University, Fiji for giving their permission to conduct this research. Besides that, we also express our gratitude to all rugby and soccer players who participated in this study.

\section{REFERENCES}

[1] Ekblom B. Applied Physiology of Soccer. Sport Med. 1986;3(1).

[2] Chaouachi A, Manzi V, Wong DP, Chaalali A, Laurencelle L, Chamari $\mathrm{K}$, et al. Intermittent endurance and repeated sprint ability in soccer players. J Strength Cond Res. 2010;24(10).

[3] Johnston W, Duignan C, Coughlan GF, Caulfield B. Dynamic balance performance varies by position but not by age group in elite Rugby Union players-a normative study. J Sports Sci. 2019;37(11).

[4] Alentorn-Geli E, Myer GD, Silvers HJ, Samitier G, Romero D, Lázaro-Haro C, et al. Prevention of non-contact anterior cruciate ligament injuries in soccer players. Part 1: Mechanisms of injury and underlying risk factors. Knee Surgery, Sport Traumatol Arthrosc. 2009;17(7).

[5] Ahsan M, Mohammad A. effects of different warm-up techniques on dynamic balance and muscular strength on players: a study. Eur J Phys Educ Sport Sci Vol 4, Issue 12, 2018DO - 1046827/ejpe.v0i02004 [Internet]. 2018 Oct 16; Available from: https://oapub.org/edu/index.php/ejep/article /view/2004

[6] Ringhof S, Stein T. Biomechanical assessment of dynamic balance: Specificity of different balance tests. Hum Mov Sci. 2018;58.

[7] Hartley EM, Hoch MC, Boling MC. Y-balance test performance and BMI are associated with ankle sprain injury in collegiate male athletes. J Sci Med Sport. 2018;21(7).

[8] Smith CA, Chimera NJ, Warren M. Association of Y balance test reach asymmetry and injury in Division I Athletes. Med Sci Sports Exerc. 2015;47(1).

[9] Johnston W, O'Reilly M, Duignan C, Liston M, McLoughlin $\mathrm{R}$, Coughlan GF, et al. Association of Dynamic Balance With Sports-Related Concussion: A Prospective Cohort Study. Am J Sports Med. 2019;47(1).

[10] Faigenbaum AD, Bagley J, Boise S, Farrell A, Bates N, Myer GD. Dynamic Balance in Children: Performance Comparison Between Two Testing Devices. Athl Train Sport Heal Care. 2015;7(4).

[11] Barati AH, Bagheri A, Azimi R, Darchini MA, Nik HN. Comparison balance and footprint parameters in normal and overweight children. Int J Prev Med. 2013;4(Suppl 1).

[12] Svensson M, Drust B. Testing soccer players. Vol. 23, Journal of Sports Sciences. 2005.

[13] Metaxas TI, Koutlianos NA, Kouidi EJ, Deligiannis AP. Comparative study of field and laboratory tests for the evaluation of aerobic capacity in soccer players. J Strength Cond Res. 2005;19(1). 
[14] Ahsan M, Ali MF. Relationship between maximal oxygen uptake and dynamic stability in university rugby and soccer players. Int J Hum Mov Sport Sci. 2021;9(4), pp. 704 - 711. DOI: $10.13189 /$ saj.2021.090414.

[15] Léger LA, Mercier D, Gadoury C, Lambert J. The multistage 20 metre shuttle run test for aerobic fitness. J Sports Sci. 1988;6(2).

[16] Margaria R, Aghemo P, Rovelli E. Measurement of muscular power (anaerobic) in man. J Appl Physiol. 1966;21(5).

[17] Jakovljević DK, Eric M, Jovanovic G, Dimitric G, Cupic MB, Ponorac N. EXPLOSIVE MUSCLE POWER ASSESSMENT IN ELITE ATHLETES USING WINGATE ANAEROBIC TEST. Rev Bras Med do Esporte [Internet]. 2018 Mar [cited 2021 Sep 14];24(2):107-11. Available from: http://www.scielo.br/scielo.php?script=sci_arttext\&pid=S15 17-86922018000200107\&lng=en\&tlng=en

[18] Shah NC, Lyandres O, Walsh JT, Glucksberg MR, Van Duyne RP. The physiological basis of physical education and athletics. Physiol Energy, Nutr Hum Performance; Lea Febiger. 1996;22(3).

[19] Mccurdy KW, Walker JL, Langford GA, Kutz MR, Guerrero JM, Mcmillan J. The relationship between kinematic determinants of jump and sprint performance in division $\mathrm{i}$ women soccer players. J Strength Cond Res. 2010;24(12).

[20] Wilczyński B, Hinca J, Ślęzak D, Zorena K. The relationship between dynamic balance and jumping tests among adolescent amateur rugby players. A preliminary study. Int $\mathbf{J}$ Environ Res Public Health. 2021;18(1).

[21] Shaffer SW, Teyhen DS, Lorenson CL, Warren RL, Koreerat CM, Straseske CA, et al. Y-balance test: A reliability study involving multiple raters. Mil Med. 2013;178(11):1264-70.

[22] Sargent DA. The Physical Test of a Man. Am Phys Educ Rev. 1921;26(4).

[23] Alahmri F, Alsaadi S, Ahsan M. Comparison of 3d hip joint kinematics in people with asymptomatic pronation of the foot and non-pronation controls. Malaysian $\mathrm{J}$ Med Sci. 2021;28(3).

[24] Flouris AD, Metsios GS, Koutedakis Y. Enhancing the efficacy of the $20 \mathrm{~m}$ multistage shuttle run test. Br J Sports Med. 2005;39(3).

[25] Johnson DL, Bahamonde R. Power Output Estimate in University Athletes. J Strength Cond Res. 1996;10(3).

[26] Coughlan GF, Fullam K, Delahunt E, Gissane C, Caulfield BM. A comparison between performance on selected directions of the star excursion balance test and the $\mathrm{Y}$ balance test. J Athl Train. 2012;47(4).

[27] Bressel E, Yonker JC, Kras J, Heath EM. Comparison of static and dynamic balance in female collegiate soccer, basketball, and gymnastics athletes. J Athl Train. 2007;42(1).

[28] Paillard T, Noé F, Rivière T, Marion V, Montoya R, Dupui P. Postural performance and strategy in the unipedal stance of soccer players at different levels of competition. J Athl Train. 2006;41(2).

[29] Pollock K. The Star Excursion Balance Test as a Predictor of Lower Extremity Injury in High School Football Players. In 2010.
[30] Butler RJ, Southers C, Gorman PP, Kiesel KB, Plisky PJ. Differences in soccer players' dynamic balance across levels of competition. J Athl Train. 2012;47(6).

[31] Ahsan M. Effects of different types of warm-up on dynamic stability and isokinetic strength. Saudi J Sport Med. 2020;20(3).

[32] Sarmento H, Marcelino R, Anguera MT, CampaniÇo J, Matos N, Leit Ão JC. Match analysis in football: a systematic review. J Sports Sci. 2014;32(20).

[33] Biological performance of German soccer players (professionals and juniors) tested by special ergometry and treadmill methods. In: Science and Football (Routledge Revivals). 2021.

[34] Saltin B, Astrand PO. Maximal oxygen uptake in athletes. J Appl Physiol [Internet]. 1967;23(3):353-8. Available from: https://doi.org/10.1152/jappl.1967.23.3.353

[35] Tønnessen E, Hem E, Leirstein S, Haugen T, Seiler S. Maximal aerobic power characteristics of male professional soccer players, 1989-2012. Int J Sports Physiol Perform. $2013 ; 8(3)$.

[36] Ahmaidi S, Collomp K, Caillaud C, Prefaut C. Maximal and functional aerobic capacity as assessed by two graduated field methods in comparison to laboratory exercise testing in moderately trained subjects. Int J Sports Med. 1992;13(3).

[37] Bellar D, Hatchett A, Judge LW, Breaux ME, Marcus L. Herthe relationship of aerobic capacity, anaerobic peak power and experience to performance in CrossFit exercise. Biol Sport. 2015;32(4).

[38] Withers RT, Sherman WM, Miller JM, Costill DL. Specificity of the anaerobic threshold in endurance trained cyclists and runners. Eur J Appl Physiol Occup Physiol. 1981;47(1).

[39] Gabbett TJ. Physiological characteristics of junior and senior rugby league players. Br J Sports Med. 2002;36(5).

[40] Da Silva CD, Bloomfield J, Marins JCB. A review of stature, body mass and maximal oxygen uptake profiles of U17, U20 and first division players in Brazilian soccer. Vol. 7, Journal of Sports Science and Medicine. 2008.

[41]Relationship between maximal aerobic power and performance of a professional soccer team. In: Science and Football II. 2021.

[42] Al-Hazzaa HM, Almuzaini KS, Al-Refaee SA, Sulaiman MA, Dafterdar MY, Al-Ghamedi A, et al. Aerobic and anaerobic power characteristics of Saudi elite soccer players. J Sports Med Phys Fitness. 2001;41(1).

[43] Wadley G, Le Rossignol P. The relationship between repeated sprint ability and the aerobic and anaerobic energy systems. J Sci Med Sport. 1998;1(2).

[44] Aziz AR, Chia M, Teh KC. The relationship between maximal oxygen uptake and repeated sprint performance indices in field hockey and soccer players. J Sports Med Phys Fitness. 2000;40(3).

[45] Minahan C, Chia M, Inbar O. Does power indicate capacity? 30-S wingate anaerobic test vs. maximal accumulated O2 deficit. Int J Sports Med. 2007;28(10). 